\title{
The Dependence of
}

\section{Ammonium-Nitrate Fuel-Oil (ANFO) Detonation on Confinement}

\author{
Scott I. Jackson \\ Shock and Detonation Physics Group \\ Los Alamos National Laboratory, Los Alamos, NM 87545 USA
}

\begin{abstract}
As detonation is a coupled fluid-chemical process, flow divergence inside the detonation reaction zone can strongly influence detonation velocity and energy release. Such divergence is responsible for the diameter-effect and failure-diameter phenomena in condensed-phase explosives and particularly dominant in detonation of nonideal explosives such as Ammonium Nitrate and Fuel Oil (ANFO). In this study, the effect of reaction zone flow divergence on ANFO detonation was explored through variation of the inert confinement and explosive diameter in the rate-stick geometry with cylinder expansion experiments. New tests are discussed and compared to prior experiments. Presented results include the detonation velocity as a function of diameter and confinement, reaction zone times, detonation product isentropes and energies, as well as sonic surface pressures and velocities. Product energy densities and isentropes were found to increase with detonation velocity, indicating more complete chemical reaction with increased detonation velocity. Detonation reaction zone times were found to scale with the acoustic transit time of the confiner wall and used to show that the ANFO diameter effect scaled with the reaction zone time
\end{abstract}


for a particle along the flow centerline, regardless of the confinement. Such a result indicates that the ANFO reaction mechanisms are sufficiently slow that the centerline fluid expansion timescale is a limiting factor controlling detonation velocity and energy release.

Key words: Detonation, Cylinder Test, Equation of State Topic: Detonations, Explosions, and Supersonic Combustion

\section{Introduction}

Detonation is a combustion mode where adiabatic shock compression provides the activation energy necessary to convert a metastable reactant mixture to a product state of lower chemical potential energy. In order to be self-sustaining, the shock compression is also supported by a portion of the released chemical energy. Thus, detonation is a coupled fluid-chemical process where the fluid dynamics of the reacting flow can significantly affect the chemical energy release.

For one-dimensional (1D) flow of a reactant mixture at a given thermodynamic state, this coupling results in a specific detonation velocity $D_{0}$ and product state. The Chapman-Jouguet (CJ) criteria can be used to approximate $D_{0}$ when the reactants and products each have a known equation of state (EOS). The ZND detonation model can also be used to further resolve the thermodynamic conditions in the detonation reaction zone for mixtures where the reaction mechanisms and intermediate EOSs are known. These models work well to predict the properties of gaseous explosives confined by rigid boundaries.

For many mixtures, however, the detonation process is more complex. Pressures $P$ in the reaction zone of condensed-phase high explosives (HEs) exceed the yield strength of most confining materials. The resulting confiner deformation laterally expands the reacting flow inside the reaction zone, decreasing pressure and temperature. Thus, reaction zone flow divergence can quench or lower the rate of chemical reactions, resulting in a partially reacted product state with decreased $D_{0}$, product energy $e$, and reaction zone length [1]. Detonation that exhibits strong sensitivity to such reaction zone flow divergence is referred to as nonideal, as its performance parameters $\left(D_{0}\right.$ and $e$ ) vary significantly from those of an idealized 1D detonation. Examples of nonideal detonation can be seen 
in the diameter-effect plot in Fig. 1 of Jackson and Short [1] for Ammonium-Nitrate and Fuel-Oil (ANFO), as well as for Comp B and TNT near their failure diameters, where $D_{0}$ is a strong function of radius. In contrast, nitromethane exhibits ideal behavior, with minimal change in $D_{0}$ from the CJ condition to the failure diameter.

Prediction of this effect via direct numerical simulation is challenging. The disparity between the smallest reaction zone scales $(10 \mu \mathrm{m})$ and the largest engineering scales (meters) precludes the possibility of adequately resolving the reaction zone on most computing resources. Even were this possible, the reaction zones of HEs consist of multiphase flow initially composed of complex reactant molecules with poorly understood chemical reaction pathways and EOSs. Experimental characterization of these properties has proved difficult as the reaction zone is at extremely high pressure (>1-50 GPa), optically opaque, and evolving rapidly. As a result, current modeling approaches [2-4] for HEs involve simplified engineering models whose calibrations rely heavily on experimental measurements at different scales. Due to the simplified model physics, prediction of the rapid variations in nonideal detonation properties with charge confinement and scale remains challenging for most existing models. Measurements of nonideal detonation parameters such as $D_{0}$, reaction zone length, reaction zone pressure, and their variation with reaction zone flow divergence are thus critical for the development of more robust HE models, which are required for both industrial and defense applications due to increased interest in nonideal HEs [5], which are used extensively in mining and also in some defense applications.

In this work, we analyze nonideal detonation of ANFO in an experimental axisymmetric rate-stick geometry using new copper cylinder expansion (CYLEX) tests and prior aluminum ones [6, 7]. The thickness and density of the inert confining tubes, as well as the HE charge diameter, were varied to alter the confiner deformation rate and flow divergence inside the detonation reaction zone. We report the effect of confinement on $D_{0}$, reaction zone sonic state conditions, $e$, and the product EOS (in isentrope form) for each test. 


\begin{tabular}{|c|c|c|c|c|c|c|c|c|c|c|c|c|c|}
\hline Test & Wall & ID & $W$ & $L / \mathrm{ID}$ & $M$ & $\rho_{e}$ & $D_{0} \pm \mathrm{SE}$ & $v_{\infty}$ & $a_{0}$ & $\omega$ & $S$ & e & Ref. \\
\hline Identifier & & $(\mathrm{mm})$ & $(\mathrm{mm})$ & & & $(\mathrm{g} / \mathrm{cc})$ & $(\mathrm{km} / \mathrm{s})$ & & $\times 10$ & $\times 10$ & $(\%)$ & $(\mathrm{kJ} / \mathrm{g})$ & \\
\hline $2-0200$ & $\mathrm{Cu}$ & 50.8 & 5.08 & 12.0 & $\times 4.56$ & 0.864 & $\times 3.224 \pm 0.008$ & 1.00 & 1.00 & 7.92 & 0.5 & 1.54 & \\
\hline $3-0250$ & $\mathrm{Al}$ & 76.2 & 6.35 & 12.0 & - 1.14 & 0.855 & $2.936 \pm 0.006$ & 1.79 & 1.59 & 8.09 & 1.5 & 1.24 & {$[7]$} \\
\hline $3-0375$ & $\mathrm{Al}$ & 76.2 & 9.53 & 12.0 & 1.85 & 0.822 & $3.089 \pm 0.014$ & 1.32 & 0.93 & 9.39 & 1.1 & 1.34 & {$[7]$} \\
\hline $3-0500$ & $\mathrm{Al}$ & 76.2 & 12.7 & 12.0 & 2.35 & 0.895 & $3.569 \pm 0.019$ & 1.23 & 0.89 & 9.59 & 1.8 & 1.62 & {$[7]$} \\
\hline 3-0501 & $\mathrm{Al}$ & 76.2 & 12.7 & 12.0 & 2.35 & 0.895 & $3.619 \pm 0.006$ & 1.41 & 1.19 & 8.25 & 2.2 & 1.73 & {$[6]$} \\
\hline $3-1000$ & $\mathrm{Al}$ & 76.2 & 25.4 & 12.0 & 5.52 & 0.870 & $3.949 \pm 0.007$ & 1.25 & 0.99 & 5.71 & 2.8 & 1.94 & {$[7]$} \\
\hline $3-2000$ & $\mathrm{Al}$ & 76.2 & 50.8 & 12.0 & 13.8 & 0.870 & $4.088 \pm 0.013$ & 0.40 & 0.33 & 8.51 & 0.5 & 1.60 & {$[7]$} \\
\hline $4-0400$ & $\mathrm{Cu}$ & 101.6 & 10.16 & 12.0 & $\times 4.60$ & 0.876 & $\times 3.865 \pm 0.010$ & 1.05 & 0.90 & 7.63 & -0.1 & 1.86 & {$[7]$} \\
\hline $4-0500$ & $\mathrm{Al}$ & 101.6 & 12.7 & 16.0 & $\diamond 1.73$ & 0.857 & $>4.114$ & 1.59 & 1.31 & 8.44 & 0.8 & 1.72 & \\
\hline 6-0500 & $\mathrm{Al}$ & 152.4 & 12.7 & 16.0 & А 1.12 & 0.873 & $\triangle 4.085 \pm 0.006$ & 1.85 & 1.66 & 8.37 & 0.1 & 1.60 & {$[7]$} \\
\hline $6-1000$ & $\mathrm{Al}$ & 152.4 & 25.4 & 16.0 & А 2.43 & 0.865 & $\Delta 4.275 \pm 0.009$ & 1.66 & 1.30 & 6.65 & -0.3 & 2.06 & {$[7]$} \\
\hline $6-2000$ & $\mathrm{Al}$ & 152.4 & 50.8 & 16.0 & $\triangle 5.54$ & 0.866 & $\Delta 4.390 \pm 0.010$ & 1.12 & 0.29 & 8.21 & 2.0 & 2.31 & {$[7]$} \\
\hline
\end{tabular}

Table 1

Summary of wall expansion tests. Parameters ID, $W, L, M, \rho_{e}$, SE, $S$ and $e$ denote the inner diameter, wall thickness, tube length, wall-to-ANFO mass ratio, ANFO initial density, velocity standard error, difference between experimental and predicted Rayleigh line slopes, and the specific energy of the products, respectively. Densities for $\mathrm{Al}$ and $\mathrm{Cu}$ were 2.70 and $8.96 \mathrm{~g} / \mathrm{cc}$, respectively. Test 3-0501 was labelled as 12-914 in Jackson et al. [6]; here $D_{0}$ is refit disregarding the first four pins, which were in the unsteady initiation region $(\approx 4 \mathrm{ID}$ from the booster), yielding a lower $\mathrm{SE}(0.006$ instead of $0.040 \mathrm{~mm} / \mu \mathrm{s})$.

\section{Current and Prior Experiments}

The experimental data utilized in this analysis is summarized in Tab. 1. It consists of a series of confined ANFO rate sticks where the expansibn velocity-time history of the inert confiner was 
recorded with a Photon Doppler Velocimetry (PDV) diagnostic. Previous tests were fielded with 6061 aluminum (Al) confiners to characterize the effect of the higher aluminum sound speed on $D_{0}[6,7]$. We also present two new CYLEX tests fielded with annealed OHFC copper $(\mathrm{Cu})$. The combination of tests exhibits a range of $M$, the ratio of the wall mass to that of the HE (Tab. 1), that are used to represent the degree of confinement.

The two copper CYLEX tests, 2-0200 and 4-0400, were scaled variants of the 25.4-mm LANL standard [8] with dimensions listed in Tab. 1. The ANFO was prepared with identical ingredients, formulation and methodology to prior work $[6,7]$ in a mass ratio of $94 \%$ HE-grade ammonium nitrate to $6 \%$ diesel fuel oil. Each copper cylinder was boosted with a 25.4-mm thick disc of PETNbased sheet explosive, which had identical outer diameter (OD) to each cylinder's inner diameter (ID) and was contained in plastic as in Short and Jackson [7].

Wall expansion velocities $v(t)$ were measured with PDV probes initially oriented normal to the wall and located approximately one-third of the total cylinder length from the downstream end. Shorting wires (as in Jackson [8]) measured $D_{0}$ from a linear least squares fit to wire trigger time and position. However, the wires did not trigger for test 4-0400, so $D_{0}$ was derived from the initial motion detected by two PDV probes located $100 \mathrm{~mm}$ axially apart, which did not yield a standard error.

\section{Experimental Results}

The measured $D_{0}$ from each test are listed in Tab. 1 and plotted in Fig. 1 versus inverse inner radius $R_{i}$ for $M$ binned into six groups. Fits to the Eyring equation (Fig. 1) are also shown. Increasing $M$ is seen to both increase $D_{0}$ for a given $R_{i}$ as well as the implied critical diameter $R_{c}$ for each curve relative to the unconfined case. Thus, increased confinement is seen to decrease the reaction zone flow divergence and increase $D_{0}$.

The $v(t)$ traces from the copper tests are shown in Fig. 2. Both tests show wall motion characteristic of subsonic wall acceleration [7]. Initial motion is smooth and absent of the discontinuous acceleration or compressible ringing generally observed in CYLEX tests $[8,9]$. The $v(t)$ profiles are fit to an analytic form [10] (shown in Fig. 2 with fit parameters in Tab. 1), that captures the $v(t)$ 


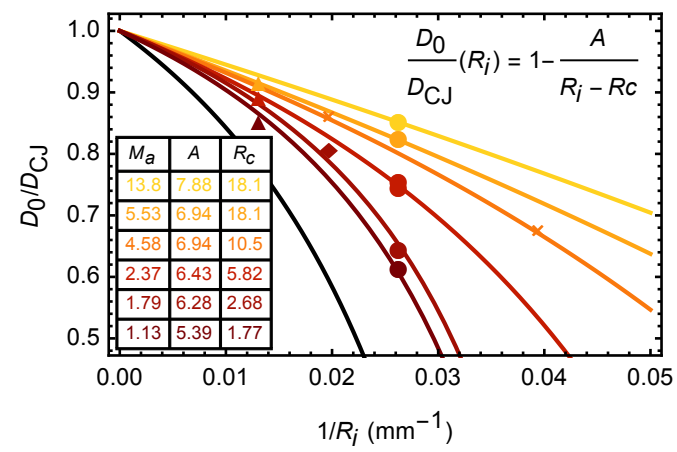

Fig. 1. Diameter effect versus $M$ with colors and symbols for $M$ from Tab. $1 . M_{a}$ is the average $M$ value for each group. Unconfined (black) curve from Ref. 1. $D_{C J}$ is specified as $4.8 \mathrm{~mm} / \mu$ s from Jackson and Short [1].

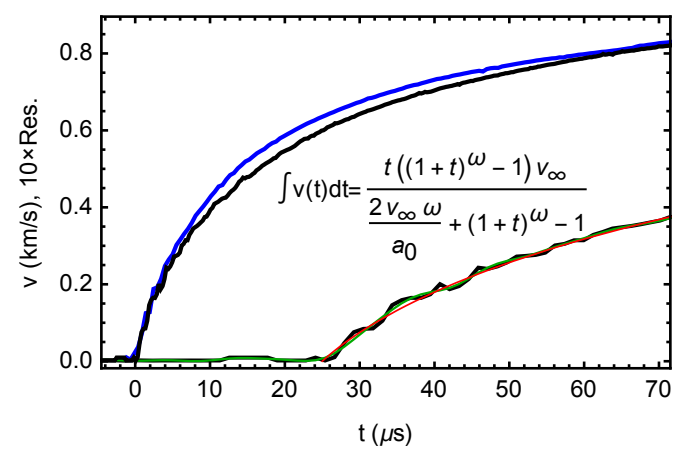

Fig. 2. The $v(t)$ data for the copper tests: 2-0200 (blue) and 4-0400 (black). Repeated trace 4-0400 is shifted by $25 \mu \mathrm{s}$, stretched in time and overlaid with its low-pass filtered form (green) and analytic fit (red). profiles well, with the exception of the smooth initial acceleration.

\section{Inferred Thermodynamic Properties}

In detonation theory, the shock compresses the reactant along the reactant Hugoniot to its intersection with the Rayleigh line. This point is referred to as the von Neumann state. Chemical reaction in the reaction zone then decreases pressure along this same Rayleigh line as the flow evolves towards a final product state. At the end of the reaction zone, the product isentrope associated with this final state intersects the Rayleigh line. For stable detonations, this reaction zone end flow is assumed to be locally sonic and isolated from the downstream. In this case, the product hugoniot and product isentrope are tangent to the Rayleigh line at the reaction zone end. In the CYLEX geometry, products then expand along the product isentrope, which diverges from the Rayleigh line with decreasing 
pressure. From conservation of mass and momentum, the Rayleigh line is $P /\left(\nu_{e}-\nu\right)=\left(\rho_{e} D_{0}\right)^{2}$, where $\nu=1 / \rho$ is the specific volume. For HEs, reactant hugoniots generally fit well to the empirical relation $D_{0}=c_{0}+s u_{p}$, where $u_{p}$ is the post-shock particle velocity and $c_{0}$ and $s$ are fit parameters. Product isentropes are fit to the JWL EOS from CYLEX data [2].

We apply a recently developed analytical CYLEX analysis [9] to infer the thermodynamic flow properties from the wall motion. This analysis solves for the full cylinder motion from the single PDV trace, infers the flow pressure from the wall acceleration, and the flow specific volume from the confiner expansion. As the confiner motion for all tests is subsonic and incompressible, the present analysis uses the incompressible approach of Jackson [9] with a single correction discussed in the Appendix. Input traces must be smooth and free of spurious noise since the analysis infers pressure from $d v / d t$. The analytic fits are thus used in place of the raw $v(t)$ data in the analysis. The analytic form does not capture the smooth initial cylinder acceleration, however. Thus, we also analyze the raw $v(t)$ data after processing it with a low-pass filter to remove noise. Specifically, low-pass filtering was implemented via the "LowpassFilter" function in Mathematica with function parameters such that (a) the product of the lowpass-cutoff frequency and velocity-record frequency was two and (b) the filter kernel length was 5000 points. (The sample frequencies of the velocity records were between 12-200 MHz.) Both smoothing approaches are illustrated for test 4-0400 in Fig. 2 and typical of the smoothed fit quality for the dataset.

The results for 2-0200 are shown in Fig. 3 for analytically fit and low-pass filtered forms of $v(t)$. Detonation arrival consists of a pressure increase to $3.5 \mathrm{GPa}$ over $2.5 \mu \mathrm{s}$ with an associated decrease

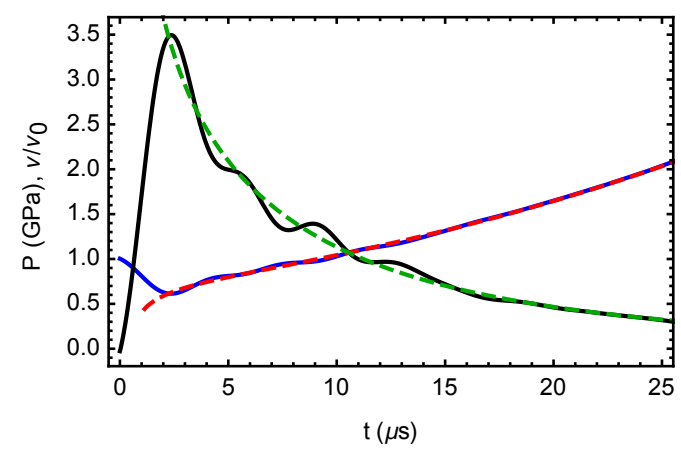

Fig. 3. $P$ and $\nu$ inferred from test 2-0200. Black and blue curves are $P$ and $\nu$ from the filtered $v(t)$ trace. Green and red curves are $P$ and $\nu$ from the analytic $v(t)$ fit. 
in $\nu$. This diffuse shock is consistent with prior studies $[6,7,11]$ which have shown that higher sound speed confinement acts to temporally disperse the detonation shock information. After peaking, $P$ decreases and $\nu$ increases for the rest of the test time. Parametrically plotting $P(t)$ versus $\nu(t)$ in Fig. 4 yields an apparent Rayleigh line associated with the dispersed shock, that is followed by a

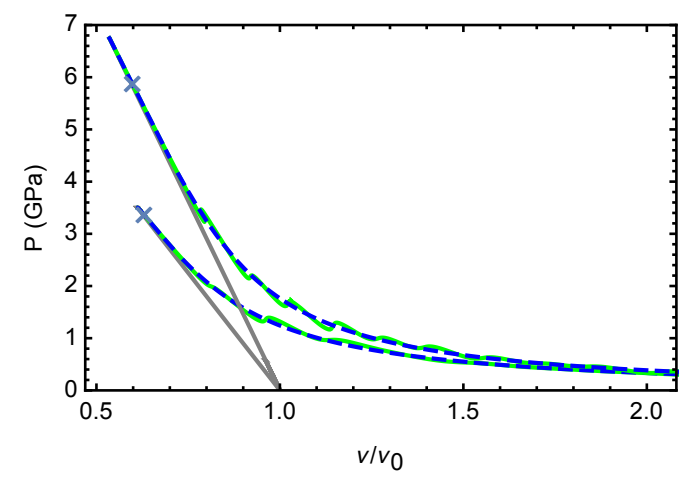

Fig. 4. Experimentally measured $P(\nu)$ evolution for copper tests 2-0200 (lower curve) and 4-0400 (higher curve). Gray lines denote data from first motion to peak pressure for the filtered $v(t)$. Blue and green curves are data from after the peak pressure for the analytic and filtered $v(t)$ forms, respectively. Crosses indicate the inferred $P$ and $\nu$ at the end of the reaction zone.

release curve, which initially travels back down the Rayleigh line before diverging onto the product isentrope. Small pressure oscillations appear on the product isentropes from the filtered $v(t)$ due to noise that was not fully removed. Thus, for the remainder of the manuscript, we use data derived from a composite $v(t)$ form that consists of the low-pass filtered trace until shortly after the peak pressure is achieved, at which point the analytic fit is used.

Direct measurement of a Rayleigh-line-like feature is unexpected, as the shock should travel along the reactant hugoniot. However, experimentally observed Rayleigh line slopes closely match the theoretical slope $\left(\rho_{e} D_{0}\right)^{2}$ predicted from the conservation equations with percent differences shown by $S$ in Tab. 1. Flow divergence during the shock-up process is thought to be responsible for the positive trend of $S$; the Rayleigh line does not account for this effect. Furthermore, while the ANFO reactant EOS has not been measured, a synthetic ANFO EOS [12] predicts higher von-Neumann pressure than is experimentally observed. This discrepancy may be due to our use of the lowpass filter. Based on these observations, we conclude that the present analysis (1) does not resolve the shock-up along the Hugoniot, but only the shock dispersion due to the complex confiner/HE 
interaction [11], (2) but does partially resolve the reaction zone relaxation down the Rayleigh line and (3) fully resolves the subsequent isentropic product expansion.

\subsection{Sonic Surface Parameters}

Observation of the reaction zone end is extremely useful to infer the reaction zone time and sound speed. This data is usually obscured by a confiner shock $[8,9]$; the subsonic confinement preserves it in this case. We identify the reaction zone end time as where the $P(\nu)$ release profile diverges from the measured Rayleigh line by $0.5 \%$ of the peak pressure. We then define the reaction zone start as the shock arrival time and the total reaction zone time as the difference between these two times. The reaction zone time at the wall $t_{w}$, measured in this fashion, is shown in Fig. 5 versus twice the transit time for an acoustic wave through the wall $2 W / c_{w}$. Reasonable agreement is observed to an

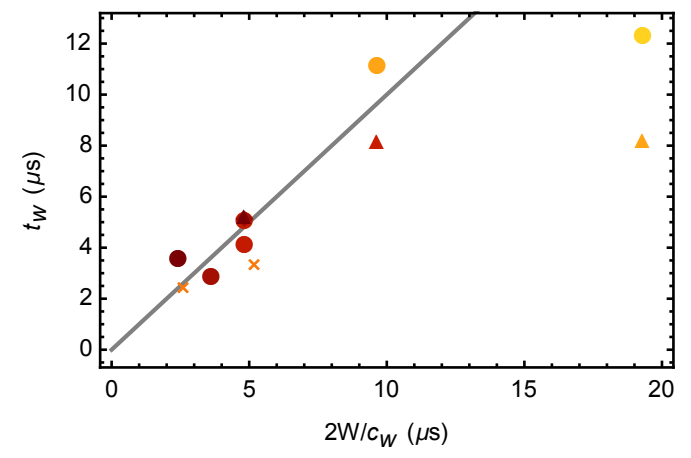

Fig. 5. Reaction zone timescale at the wall versus twice the confiner acoustic time with $c_{w}$ as 5.266 and $3.927 \mathrm{~mm} / \mu$ s for aluminum and copper, respectively. The gray line has a slope of unity.

approximate upper limit of $10 \mu \mathrm{s}$. Additionally, the reaction zone timescale is, on average, only $7 \%$ greater than the time between shock arrival and onset of peak pressure.

We interpret this correlation to indicate that inner wall motion expands the flow near the wall and induces the onset of the sonic surface that terminates the reaction zone, as described below. The pressure increase associated with detonation arrival drives a compression wave outwards into the confiner wall. For subsonic confiner motion, the wall will initially only compress minimally elastically, but will not begin large-scale plastic deformation until this compression wave reflects from the outer radius as an expansion wave. Significant expansion and pressure decrease of the reaction zone, which we postulate ends the reaction zone, only occurs after arrival of the expansion 
wave at the inner wall radius. For incompressible confiner motion, these acoustic waves travel near the confiner sound speed $c_{w}$, yielding a round trip time of $2 W / c_{w}$. If correct, this understanding would indicate that the dominant effect of the confiner thickness is to limit significant flow expansion and prevent premature onset of the sonic surface. In the present study, this effect appears to plateau near $10 \mu \mathrm{s}$, after which further increase in $W$ has a limited effect. Also, we note this technique only infers the flow conditions at the inner surface of the confiner, while axisymmetric detonations can exhibit significant variation in properties along their radius [3], with the shortest reaction zone times and lowest pressure at the charge edge. Thus, the reported $t_{w}$ are likely minimum effective values for the flow. We address reaction zone times at the charge centerline below.

\subsection{Isentrope Variation with Wall Thickness}

Figure 6 plots the measured product isentropes, from the inferred sonic surface condition to $\nu / \nu_{0}$ $=5$, for each charge ID. Higher values of $M$ (as indicated by lighter curve coloring) result in higher product isentropes for each ID and are indicative of more complete chemical reaction. The smallest IDs have reaction zone end (or sonic surface) pressures below $3 \mathrm{GPa}$, while the larger diameters have sonic surface pressures between 3.4-5.9 GPa, which are consistent with the current estimated ANFO CJ pressure of 4.5-6.3 GPa [10]. Isentropes associated with the larger ID tests access higher pressure and exhibit a decrease in slope above $3 \mathrm{GPa}$ on the log-log plots, indicating the existence of a more compressible state than at lower pressure, which is an unusual feature. All curves are below the CJ product isentrope predicted by the thermochemical code CHEETAH [13], with a single exception as discussed below. Product isentropes were fit to the JWL EOS [2]

$$
P(\nu)=\Lambda_{1} e^{-\lambda_{1} \frac{\nu}{\nu e}}+\Lambda_{2} e^{-\lambda_{2} \frac{\nu}{\nu_{e}}}+\Lambda_{3}{\frac{\nu}{\nu_{e}}}^{-\left(\lambda_{3}+1\right)}
$$

with fit parameters listed in Table 2 .

Two tests with the largest $W$ also exhibit anomalous behavior relative to the trend. The $P(\nu)$ trajectory for test 3-2000 (lightest yellow curve in Fig. 6a) drops more quickly than the other traces. We interpret that this test (with $M$ of 14!) had a thick enough wall to violate the negligible confiner deformation stress assumption of our model [9]. Thus, significant product energy is being 

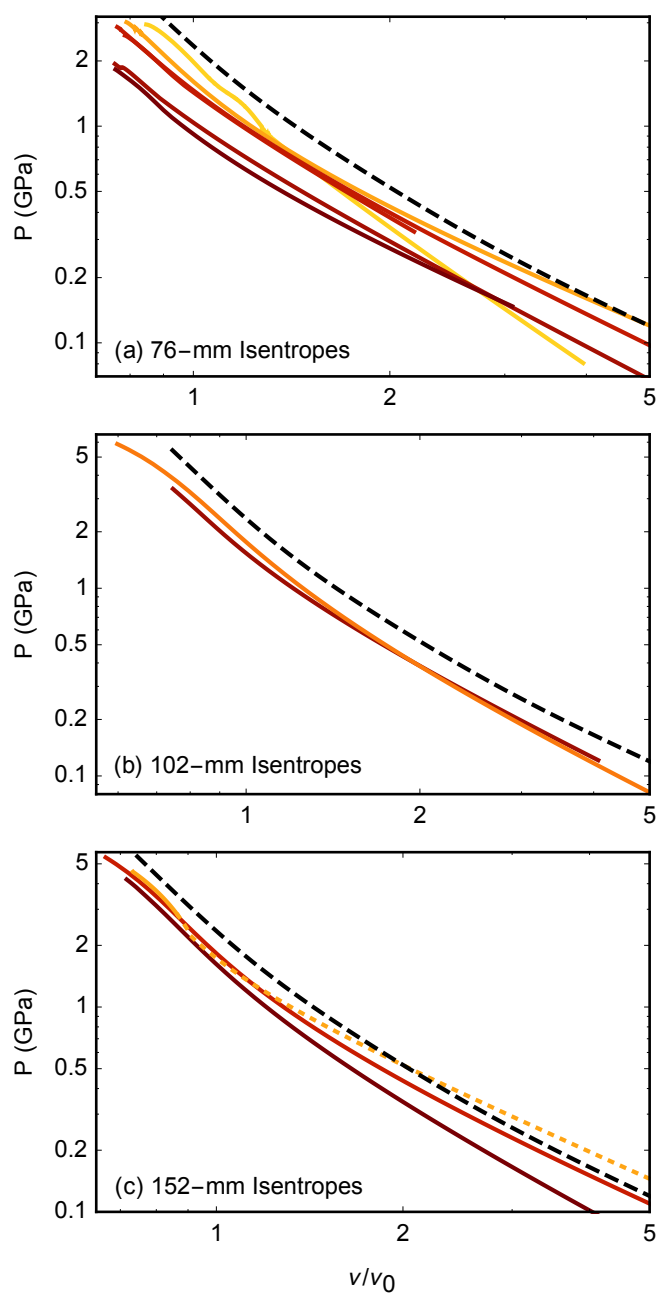

Fig. 6. Isentropes for each ID using colors for $M$ from Tab. 1 symbols. The dashed black curve is the predicted product isentrope for a CJ (1D) detonation [13].

lost to plastic wall deformation and is not captured by our analysis. This effect could be further explored numerically with a strain-rate dependent model, though calibration data at these high strain rates are scarce. The confiner strength could also be varied more significantly, through use of different alloys or annealing levels, use of plastics and liquids, or use of geometries that induce less confiner strain. Test 6-2000 (lightest curve in Fig. 6c, with $M=5.5$ ) is believed to have ruptured early (see the discontinuous acceleration near $16 \mu$ s in Fig. 13 of Short and Jackson [7]), data derived after this time is suspect and plotted with a dotted line. At late expansion, the suspect portion of the curve crosses the predicted CJ isentrope. Additional dynamics associated with the coupled HE / wall interaction [11] may contribute to the anomalous expansion for these two thicker-wall tests, however, repeat experiments with additional diagnostics (such as framing cameras and multiple 
PDV probes) would be necessary to explore this issue further.

In lieu of additional experimentation, use of dense, ductile, and thin confiners is recommended to avoid such strength and fragmentation issues. The present data set for copper is limited and interpretation of the PDV spectra is not definitive, but the average tube diameter at failure onset was estimated to be approximately $130 \%$ and $310 \%$ of the initial diameter for aluminum and copper confiners, respectively. In evaluating these values, wall "failure onset" was interpreted to be indicated by a discontinuous acceleration in wall velocity (indicating a sudden change in wall strength) or sudden broadening of the PDV spectra (indicating wall fragmentation). These results are consistent with the relative quasi-static elongation at break values for aluminum 6061-T6 and annealed copper. Note that onset of wall failure does not necessarily indicate the loss of product confinement, which yields a different PDV spectra.

\subsection{Available Product Energy Variation with $D_{0}$}

Thermochemical calculations for ANFO with end products of $\mathrm{CO}_{2}, \mathrm{H}_{2} \mathrm{O}, \mathrm{N}_{2}$, and $\mathrm{O}_{2}$ overestimate $D_{0}$ and $e$, indicating that $\mathrm{ANFO}$ detonations produce an intermediate product state of incomplete combustion. The difference between the areas under the product isentrope and Rayleigh line in $P(\nu)$ space yields the product energy $e$ available for expansion work and provides a relative measure of the degree of chemical reaction for each test. Integrating each product isentrope in this fashion between $\nu=7 \mathrm{~g} / \mathrm{cc}$ and the sonic surface yields $e$ as plotted in Fig. 7 . The trend indicates that increasing $D_{0}$

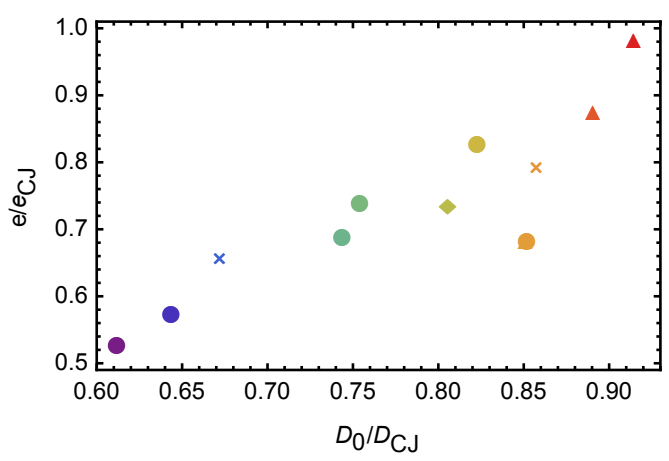

Fig. 7. The product energy versus $D_{0} / D_{C J}$ with $e_{C J}=2.35 \mathrm{~kJ} / \mathrm{g}$ [13]. Colors and symbols for $D_{0}$ from Tab. 1.

generates higher $e$ and, thus, more complete reaction. Plotting all of the measured product isentropes 


\begin{tabular}{|c|c|c|c|c|c|c|c|c|}
\hline Test & $\Lambda_{1}$ & $\Lambda_{2}$ & $\Lambda_{3}$ & $\lambda_{1}$ & $\lambda_{2}$ & $\lambda_{3}$ & $\nu_{C J}$ & $P_{C J}$ \\
\hline $2-0200$ & 32.0 & 2.65 & 0.79 & 5.15 & 2.22 & 0.43 & 0.73 & 3.36 \\
\hline $3-0250$ & 166 & 2.23 & 0.62 & 7.35 & 2.41 & 0.29 & 0.89 & 1.83 \\
\hline $3-0375$ & 53.7 & 36.6 & 0.90 & 10.6 & 5.54 & 0.60 & 0.92 & 1.94 \\
\hline $3-0500$ & 46.0 & 97.5 & 1.26 & 8.00 & 6.32 & 0.71 & 0.88 & 2.62 \\
\hline 3-0501 & 294 & 3.69 & 1.00 & 7.65 & 2.43 & 0.45 & 0.85 & 2.87 \\
\hline $3-1000$ & 634 & 7.33 & 0.86 & 8.19 & 2.56 & 0.23 & 0.91 & 3.02 \\
\hline $3-2000$ & -3158 & 165 & 1.35 & 9.93 & 5.33 & 1.05 & 0.97 & 2.94 \\
\hline $4-0400$ & 10.3 & 21.1 & 1.01 & 3.70 & 3.60 & 0.56 & 0.70 & 5.87 \\
\hline 4-0500 & 458 & 6.31 & 0.99 & 8.13 & 2.70 & 0.51 & 0.85 & 3.39 \\
\hline $6-0500$ & 338 & 13.0 & 0.90 & 7.74 & 3.09 & 0.59 & 0.82 & 4.19 \\
\hline $6-1000$ & 85.4 & 18.2 & 1.03 & 5.91 & 3.39 & 0.39 & 0.77 & 5.37 \\
\hline $6-2000$ & 2286 & 7.61 & 1.29 & 9.71 & 3.21 & 0.36 & 0.85 & 4.57 \\
\hline
\end{tabular}

Table 2

Fit JWL parameters with $\Lambda_{1}, \Lambda_{2}, \Lambda_{3}$ and $P_{C J}$ in GPa and $\nu_{C J}$ in cc/g.

as a function of $D_{0}$ (Fig. 8) also illustrates that higher pressure and more complete product states are consistently achieved with higher $D_{0}$, but that the predicted CJ state is never exceeded with the present data. Only test 6-2000, which experienced premature wall failure, crosses the predicted CJ product isentrope below 0.5 GPa (Fig. 8). The extra $e$ associated with this anomalous trajectory yields $e / e_{C J}=0.98$, which is high relative to the trend in Fig. 7 . 


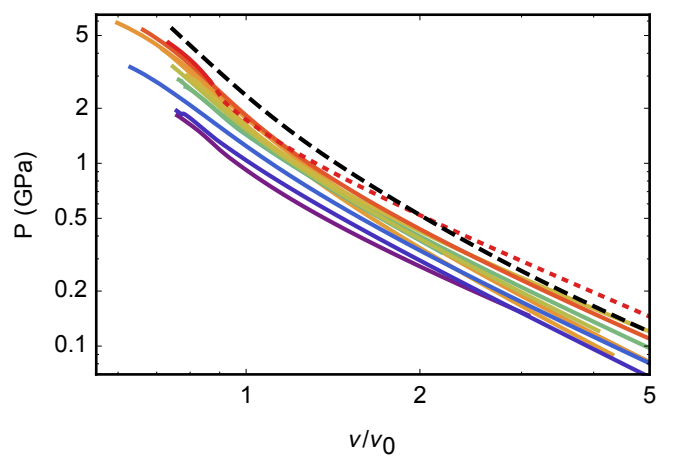

Fig. 8. All product isentropes with colors indicating $D_{0}$ from Fig. 7. Black curve is the CJ product isentrope.

\section{Reaction Time on the Flow Centerline}

It was shown above that confinement was able to increase the reaction zone time near the wall and postulated that confinement delayed the arrival of expansion waves associated with lateral flow deformation that terminated the reaction zone. While arrival of the expansion waves ended the reaction zone near the wall, it would take additional time for these waves to process the reacting flow closer to the centerline of the charge. To leading order, this additional time can be approximated by $R_{i} / c_{r z}$, where $c_{r z}$ is the reaction zone sound speed. As sound speed along a product isentrope is given by $c=\left.\sqrt{\partial P / \partial \rho}\right|_{s}$, the sonic surface condition measured for each experimental product isentrope yields the effective $c_{r z}$ for each test. Figure 9 plots $D_{0}$ versus the inverse of the time for an expansion wave to reach the charge centerline, $t_{c}=R_{i} / c_{r z}+2 W / c_{w}$, effectively replacing $R_{i}$ in Fig. 1 with $t_{c}$. The confined data is seen to collapse to a curve, indicating that the reaction zone

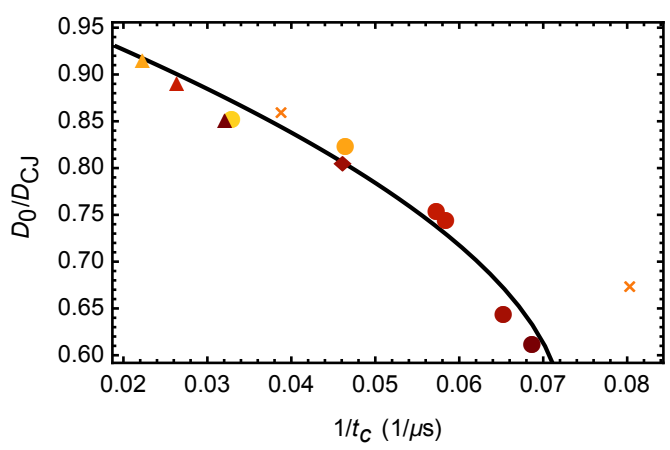

Fig. 9. Diameter effect data (symbols) from Fig. 1 rescaled by centerline reaction zone time and compared to the unconfined curve.

time along the centerline is a dominant parameter for the present experiments. The two copper tests 
are high relative to the trend, possibly indicating a secondary effect associated with wall density or its influence on expansion that is not captured with this scaling. Additional copper tests would be needed to explore this effect.

It is also desirable to similarly scale the unconfined diameter-effect curve, but no direct reaction zone measurements exist for unconfined ANFO versus charge diameter. However, Detonation Shock Dynamics (DSD) [3] calibrations measure the local normal detonation velocity $D_{n}$ as a function of the local shock curvature $\kappa$ and assume that $1 / \kappa \propto \ell_{r z}$, where $\ell_{r z}$ is the reaction zone length. The reaction zone time scaling can then be found from $t_{c}=\ell_{r z} / u_{p}=\left(\beta \kappa u_{p}\right)^{-1}$, where $\beta$ is the constant of proportionality such that $1 / \kappa \propto \beta \ell_{r z}$. With the assumption that $t_{c}$, the centerline reaction zone time, is only a function of $D_{0}, \beta$ can be found from comparison of the measured confined $t_{c}$ values to the unconfined scaling, $\left(\beta \kappa u_{p}\right)^{-1}$, using $\kappa\left(D_{n}\right)$ calibration values from the centerline. If the time for the expansion wave to reach the flow centerline is a dominant parameter on $D_{0}$ for ANFO, the shape of the unconfined diameter-effect curve should match that of the confined data when plotted as $D_{0}=f\left(1 / t_{c}\right)$. Proportionality constant $\beta$ serves only to horizontally stretch and translate the unconfined curve in a combined fashion.

To implement this analysis, we interpolate $D_{0}(\kappa)$ from a fit to the centerline $D_{n}(\kappa)$ data from Bdzil et al. [3] to find $D_{0}=4.80-148.3 \kappa+1750.8 \kappa^{2}$, valid from $D_{0}=2.45-4.80 \mathrm{~mm} / \mu \mathrm{s}$ and with $D_{0}$ in $\mathrm{mm} / \mu \mathrm{s}$ and $1 / \kappa$ in $\mathrm{mm}$. The reactant ANFO EOS of Wescott [12] yields $D_{0}=0.977+1.42 u_{p}$ with $D_{0}$ and $u_{p}$ in $\mathrm{mm} / \mu \mathrm{s}$. The subsequently computed unconfined reaction zone times are found to scale well with the confined ones for $\beta=3$.3. This magnitude of $\beta$ is consistent with the nonideal nature of ANFO, for which $\mathrm{O}(1 / \kappa) \approx \mathrm{O}\left(\ell_{r z}\right)[3]$. When the unconfined diameter-effect curve is rescaled in this fashion, its shape and location agree quite well with the confined data indicating that the centerline reaction time is a dominant parameter for $D_{0}$ in ANFO detonation. Such a result indicates that the ANFO reaction mechanisms are sufficiently slow that the centerline fluid expansion timescale $t_{c}$ is a limiting factor controlling $D_{0}$ and $e$. 


\section{Conclusions}

Cylinder expansion experiments of nonideal detonation in ANFO with varying charge diameter and confinement were used to evaluate the effect of reaction zone flow divergence on detonation velocity and detonation product energy. Increasing confinement was found to result in increased detonation velocity relative to identical unconfined charge diameters. Measured wall expansion velocities were used to infer the explosive pressure and density inside both the detonation reaction zone and products. Measured Rayleigh lines were found to be consistent with theory. For a given charge diameter, product isentropes indicated more complete chemical reaction in the detonation with increased confinement. In general, product energy densities and isentropes were found to increase with detonation velocity, also indicating more complete chemical reaction with increased detonation velocity. Detonation reaction zone times were found to scale with the acoustic transit time of the confiner wall. The diameter effect of all confined and unconfined data was found to scale well with the reaction zone time for a particle along the flow centerline. The reported thermodynamic parameters from the detonation reaction zone and products will aid in the development of accurate reaction mechanism sets and equations of state for ANFO. The study also demonstrates the utility of the cylinder expansion test to resolve the reacting flow of a detonation reaction zone when utilized with a high sound speed confiner and suitable analysis methodology.

\section{Acknowledgements}

The author acknowledges significant technical discussions on this topic with Mark Short, who was also the driving force behind the aluminum-confined ANFO experiments. The author is also grateful to Gerrit Sutherland for noting that Eq. 1 in Jackson [9] was inconsistent with the output of hydrocode computations of the CYLEX geometry. Funding for this analysis was provided by the U.S. Department of Energy's Dynamic Materials Properties ("Campaign 2") program. The copper CYLEX experiments were supported by the Department of Homeland Security. 


\section{References}

[1] S. I. Jackson, M. Short, J. Fluid Mech. 773 (2015) 224-266.

[2] E. L. Lee, H. C. Horning, J. W. Kury, Adiabatic Expansion of High Explosive Detonation Products, Technical Report UCRL-50422, Lawrence Livermore National Laboratory, Livermore, 1968.

[3] J. B. Bdzil, T. D. Aslam, R. A. Catanach, L. G. Hill, M. Short, in: 12th Det. Symp., ONR, 2002, pp. 409-417.

[4] C. Tarver, J. Hallquist, L. Erickson, in: 8th Det. Symp., ONR, 1985, pp. 951-961.

[5] D. Kennedy, Le Journal de Physique IV 5 (1995) C4-191.

[6] S. I. Jackson, C. B. Kiyanda, M. Short, Proc. Combust. Inst. 33 (2011) 2219-2226.

[7] M. Short, S. I. Jackson, Combust. Flame 162 (2015) 1857-1867.

[8] S. I. Jackson, in: 15th Det. Symp., ONR, 2015, pp. 171-180.

[9] S. I. Jackson, Proc. Combust. Inst. 35 (2015) 1997-2004.

[10] L. L. Davis, L. G. Hill, in: AIP Conf. Proc., volume 620, p. 165.

[11] M. Short, J. J. Quirk, C. B. Kiyanda, S. I. Jackson, M. E. Briggs, M. A. Shinas, in: 14th Det. Symp., ONR, 2010, pp. 769-778.

[12] B. L. Wescott, in: AIP Conf. Proc., volume 955, pp. 433-436.

[13] L. Fried, P. Souers, CHEETAH: A Next Generation Thermochemical Code, Technical Report UCRL-117240, Lawrence Livermore National Laboratory, Livermore, 1994.

\section{A Appendix: Corrected Kinematics for the Cylinder Analysis}

Equation 1 in Jackson [9] solves for the axial cylinder velocity $u$ as a function of $D_{0}$ and measured radial velocity $v$ from the PDV probe. Both components of motion are then used to reconstruct the fully two-dimensional cylinder motion rather than assuming quasi-1D flow. Equation 1 in Jackson [9] is derived from the physically inaccurate assumption that the wall material velocity vector is always normal to the wall surface in the shock-fixed frame, $\tan \theta=-u / v$. It is more physically realistic to assume that the acceleration vector is, at all times, normal to the wall surface, $\tan \theta=$ 
$-d u / d v=\frac{-d u}{d t} \frac{d t}{d v}$ as the product pressure acts normal to the cylinder interior. This constraint can be combined with that for the wall surface angle $\tan \theta=v /(D+u)$ to yield

$$
u=-D_{0}+\sqrt{D_{0}^{2}-v^{2}}
$$

Thus, this equation should replace Eq. 1 in Jackson [9]. As $u$ is generally a small component (0-5\%) of the total cylinder velocity during the high pressure (> $1 \mathrm{GPa})$ acceleration phase, this change has a negligible effect on the computed product isentrope, however it does affect the inferred wall angle, reducing it slightly at late expansion times. The other effect is to reduce the angle of the wall material velocity vector for the confiner to approximately half of the confiner wall angle. Under the prior analysis [9], it was equivalent to the wall angle. 


\section{Figure Captions}

Fig. 1: Diameter effect versus $M$ with colors and symbols for $M$ from Tab. $1 . M_{a}$ is the average $M$ value for each group. Unconfined (black) curve from Ref. 1. $D_{C J}$ is specified as $4.8 \mathrm{~mm} / \mu \mathrm{s}$ from Jackson and Short [1].

Fig. 2: The $v(t)$ data for the copper tests: 2-0200 (blue) and 4-0400 (black). Repeated trace 4-0400 is shifted by $25 \mu$ s, stretched in time and overlaid with its low-pass filtered form (green) and analytic fit (red).

Fig. 3: Experimentally measured $P(\nu)$ evolution for copper tests 2-0200 (lower curve) and 4-0400 (higher curve). Gray lines denote data from first motion to peak pressure for the filtered $v(t)$. Blue and green curves are data from after the peak pressure for the analytic and filtered $v(t)$ forms, respectively. Crosses indicate the inferred $P$ and $\nu$ at the end of the reaction zone.

Fig. 4: Experimentally measured $P(\nu)$ evolution for Cu tests 2-0200 (lower curve) and 4-0400 (higher curve). Gray lines denote data from first motion to peak pressure for the filtered $v(t)$. Blue and green curves are data from after the peak pressure for the analytic and filtered $v(t)$ forms, respectively. Crosses indicate the inferred $P$ and $\nu$ at the end of the RZ.

Fig. 5: Reaction zone timescale at the wall versus twice the confiner acoustic time with $c_{w}$ as 5.266 and $3.927 \mathrm{~mm} / \mu$ s for aluminum and copper, respectively. The gray line has a slope of unity.

Fig. 6: Isentropes for each ID using colors for $M$ from Tab. 1 symbols. The dashed black curve is the predicted product isentrope for a CJ (1D) detonation [13].

Fig. 7: The product energy versus $D_{0} / D_{C J}$ with $e_{C J}=2.35 \mathrm{~kJ} / \mathrm{g}[13]$. Colors and symbols for $D_{0}$ from Tab. 1. 
Fig. 8: All product isentropes with colors indicating $D_{0}$ from Fig. 7. Black curve is the CJ product isentrope.

Fig. 9: Diameter effect data (symbols) from Fig. 1 rescaled by centerline reaction zone time and compared to the unconfined curve. 